\title{
Two cases of non-fluent variant primary progressive aphasia with different pathological diagnoses
}

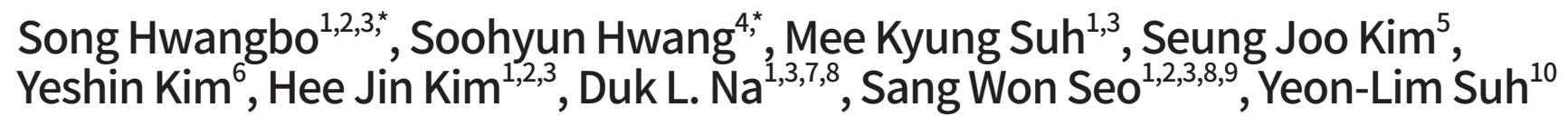

${ }^{1}$ Department of Neurology, Samsung Medical Center, Sungkyunkwan University School of Medicine, Seoul, Korea

${ }^{2}$ Samsung Alzheimer Research Center, Samsung Medical Center, Seoul, Korea

${ }^{3}$ Neuroscience Center, Samsung Medical Center, Seoul, Korea

${ }^{4}$ Department of Pathology and Translational Genomics, Samsung Medical Center, Sungkyunkwan University School of Medicine, Seoul, Korea

${ }^{5}$ Department of Neurology, Gyeongsang National University Changwon Hospital, Gyeongsang National University College of Medicine, Changwon, Korea

${ }^{6}$ Department of Neurology, Kangwon National University Hospital, Kangwon National University School of Medicine, Chuncheon, Korea

${ }^{7}$ Stem Cell \& Regenerative Medicine Institute, Samsung Medical Center, Seoul, Korea

${ }^{8}$ Department of Clinical Research Design and Evaluation, Samsung Advanced Institute for Health Sciences \& Technology,

Sungkyunkwan University, Seoul, Korea

${ }^{9}$ Center for Clinical Epidemiology, Samsung Medical Center, Seoul, Korea

${ }^{10}$ Department of Pathology, Samsung Medical Center, Sungkyunkwan University School of Medicine, Seoul, Korea

Received: March 10, 2021

Revised: April 6, 2021

Accepted: April 8, 2021

Corresponding authors:

Sang Won Seo

Department of Neurology,

Samsung Medical Center,

Sungkyunkwan University

School of Medicine, 81 Irwon-ro,

Gangnam-gu, Seoul 06351, Korea

Tel: +82-2-3410-6147

E-mail: sangwonseo@empas.com

Yeon-Lim Suh

Department of Pathology,

Samsung Medical Center,

Sungkyunkwan University

School of Medicine, 81 Irwon-ro,

Gangnam-gu, Seoul 06351, Korea

Tel: +82-2-3410-2761

E-mail:yl.suh@samsung.com

*Song Hwangbo and Soohyun Hwang contributed equally to this study as first authors.

\section{ABSTRACT}

Non-fluent variant primary progressive aphasia (nfvPPA), a subtype of frontotemporal lobar dementia syndrome, has been proven to have various pathological diagnoses. A 63-year-old woman and 71-year-old man separately visited our clinic for language dysfunction. Both patients showed non-fluent speech. The female patient showed personality change accompanied by language dysfunction, while the male patient had parkinsonian symptoms such as bradykinesia and cogwheel rigidity. Both patients were clinically diagnosed with nfvPPA. Several years after the first visit, the patients died, and a brain autopsy was performed. On postmortem examination, the female patient was pathologically diagnosed with Pick's disease, while the male patient was diagnosed with progressive supranuclear palsy. Our report suggests that nfvPPA patients might show distinct clinical features depending on underlying pathologies.

Keywords: Autopsy; Pick disease of the brain; Primary progressive nonfluent aphasia; Supranuclear palsy, progressive

This is an Open Access article distributed under the terms of the Creative Commons Attribution Non-Commercial License (https://creativecommons.org/licenses/by-nc/4.0/). 


\section{INTRODUCTION}

Frontotemporal lobar dementia (FTD) syndromes include a behavioral variant (bvFTD) and two subtypes of primary progressive aphasia [1,2]. These syndromes are united by overlapping clinical and anatomical features and their link to the underlying frontotemporal lobar degeneration pathology (FTLD) [1,2]. FTLD is characterized by neuronal and glial inclusions immunoreactive for hyperphosphorylated tau (FTLD-tau), or TAR DNA-binding protein of $43 \mathrm{kDa}$ (TDP-43, FTLD-TDP) $[3,4]$. Despite attempts to associate FTD clinical syndromes with specific FTLD neuropathological diagnoses, no such correlation has yet been proven invariant [5-7]. In particular, non-fluent variant primary progressive aphasia (nfvP$\mathrm{PA}$ ) has been proven to have various pathological diagnoses. Over $60 \%$ of nfvPPA cases show FTLD-tau, including Pick's disease (PiD), progressive supranuclear palsy (PSP), and corticobasal degeneration. Most of the remaining cases show TDP type A and Alzheimer's disease pathology $[6,8]$. Herein, we report two patients clinically diagnosed with nfvPPA that displayed different pathological findings at autopsy.

\section{CASE REPORTS}

The collection of the data was conducted as set forth in the Declaration of Helsinki. This study was approved by the Insti- tutional Review Board of Samsung Medical (IRB Rile No. 2016-11-111) and we obtained informed consent from the participant and their next of kin (daughter and spouse).

\section{Case 1}

A 63-year-old right-handed woman visited our clinic due to language dysfunction and personality change. She had started to show effortful, non-fluent speech and a decreased amount of speech 1 year prior to her first visit. After 1 year, she showed abulia and blankly watched TV all day. She occasionally got upset for no reason. There was no familial history of dementia. She had hypertension and hyperlipidemia, and was on hemodialysis for chronic renal failure.

On neurological examination, the patient showed motor impersistence and grasp reflex on the bilateral upper extremities. She scored 22 out of 30 on the Mini-Mental State Examination (MMSE). There were no signs of Parkinsonism. Detailed neuropsychological tests revealed that her attention, language, and verbal memory were decreased; especially her frontal executive function, which was severely decreased. On the Western Aphasia Battery (WAB), she demonstrated non-fluent speech and a lack of verbal response. The average number of word segments per utterance was one; word comprehension was mildly decreased, but complex sentence comprehension was remarkably decreased, confrontational naming ability was severely decreased, and repetition was

Table 1. Western Aphasia Battery (WAB) results

\begin{tabular}{|c|c|c|c|}
\hline \multirow{3}{*}{ Category of K-WAB (highest possible score) } & \multicolumn{3}{|c|}{ Raw score } \\
\hline & \multirow{2}{*}{$\begin{array}{c}\text { Case } 1 \\
2013.06 .15\end{array}$} & \multicolumn{2}{|c|}{ Case 2} \\
\hline & & 2009.12 .14 & 2011.12.07 \\
\hline Spontaneous speech (20) & 8 & 17 & 14 \\
\hline Information content (10) & 3.5 & 9 & 7 \\
\hline Fluency $(10)$ & 4.5 & 8 & 7 \\
\hline Comprehension (10) & 6.05 & 8.75 & 7.25 \\
\hline Yes/No question (3) & 1.65 & 2.85 & 2.55 \\
\hline Auditory word recognition (3) & 2.6 & 2.75 & 2 \\
\hline Sequential commands (4) & 1.8 & 3.15 & 2.55 \\
\hline Repetition (10) & 5.3 & 8.5 & 7.2 \\
\hline Naming (10) & 4.3 & 5.6 & 7.1 \\
\hline Confrontation naming (6) & 4.3 & 8.4 & 5.1 \\
\hline Generative naming (2) & 0 & 0.8 & 0.4 \\
\hline Sentence completion (1) & 0 & 1 & 0.6 \\
\hline Responsive speech (1) & 0 & 1 & 1 \\
\hline Reading (10) & & 8.2 & 4.7 \\
\hline Writing (10) & & 7.6 & 8.3 \\
\hline
\end{tabular}



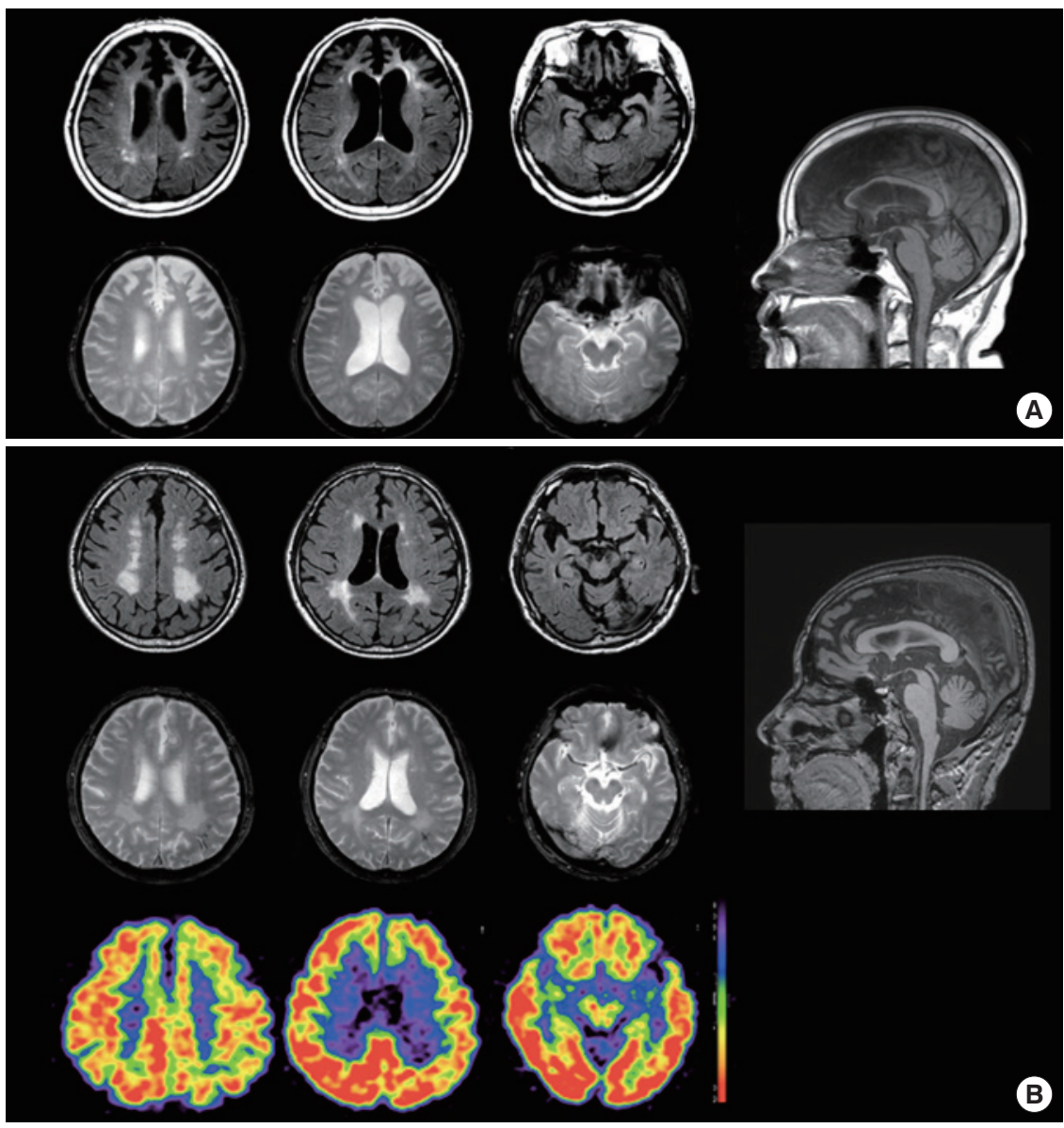

Fig. 1. Brain images of a 63-year old woman (A) and 71-year old man (B). (A) Axial fluid-attenuated inversion recovery (FLAIR) magnetic resonance imaging (MRI) showing bilateral frontal and anterior temporal atrophy. (B) Axial FLAIR MRI images showing diffuse brain atrophy and periventricular white matter hyperintensities in the anterior and posterior portion. On sagittal T1-weighted images, there is no significant midbrain atrophy. Gradient echo images showing strictly multiple lobar microbleeds and cortical superficial siderosis in the left parietal cortex. [18F]-fluorodeoxyglucose positron emission tomography showing moderate hypometabolism in the bilateral frontal cortex which is more severe on the left.

mildly decreased. In particular, she showed word-finding difficulty and delayed word retrieval. There was no effortful speech, agrammatism, or dysarthria. These results indicated a moderate degree of Broca's aphasia (Table 1). Her fluid-attenuated inversion recovery (FLAIR) images showed bilateral frontal and anterior temporal atrophy (Fig. 1). Based on her clinical symptoms, neuropsychological and language tests, and brain magnetic resonance imaging (MRI), the patient was clinically diagnosed with nfvPPA. One year after her first visit, she displayed blunted affect and did not welcome her children. Two years after her first visit, her spontaneous speech was limited to one word. She was not able to wash herself, making activities of daily living impossible without aid. Four years after her first visit, she was found dead at home. After her death, the patient's body underwent a brain autopsy at the Samsung Medical Center.
Postmortem examination showed a decreased brain weight of $988 \mathrm{~g}$ (standard for age: 1,360 g). Gross examination revealed moderate to severe gyral atrophy in the bilateral frontal lobes, and mild gyral atrophy in the bilateral parietal and medial temporal lobes (Fig. 2A). Histological examination revealed microvacuolation with severe neuronal loss and gliosis in the frontal cortex layers 2 and 3 (Fig. 2B). Mild neuronal loss and gliosis were present in the parietal cortex layer 2 , temporal cortex layer 2, amygdala, basal nucleus, basal ganglia, and dentate nucleus. Tau-positive intraneuronal cytoplasmic inclusions (Pick bodies), identified by tau and 3-repeat tau stains, were located in the frontal, parietal, and temporal cortex layers. Pick bodies were also found in the dentate gyrus, hippocampus, subiculum, basal ganglia, thalamus, periaqueductal gray matter, locus coeruleus, and pontine nucleus (Fig. 2C). In the absence of TDP-43 pathology 


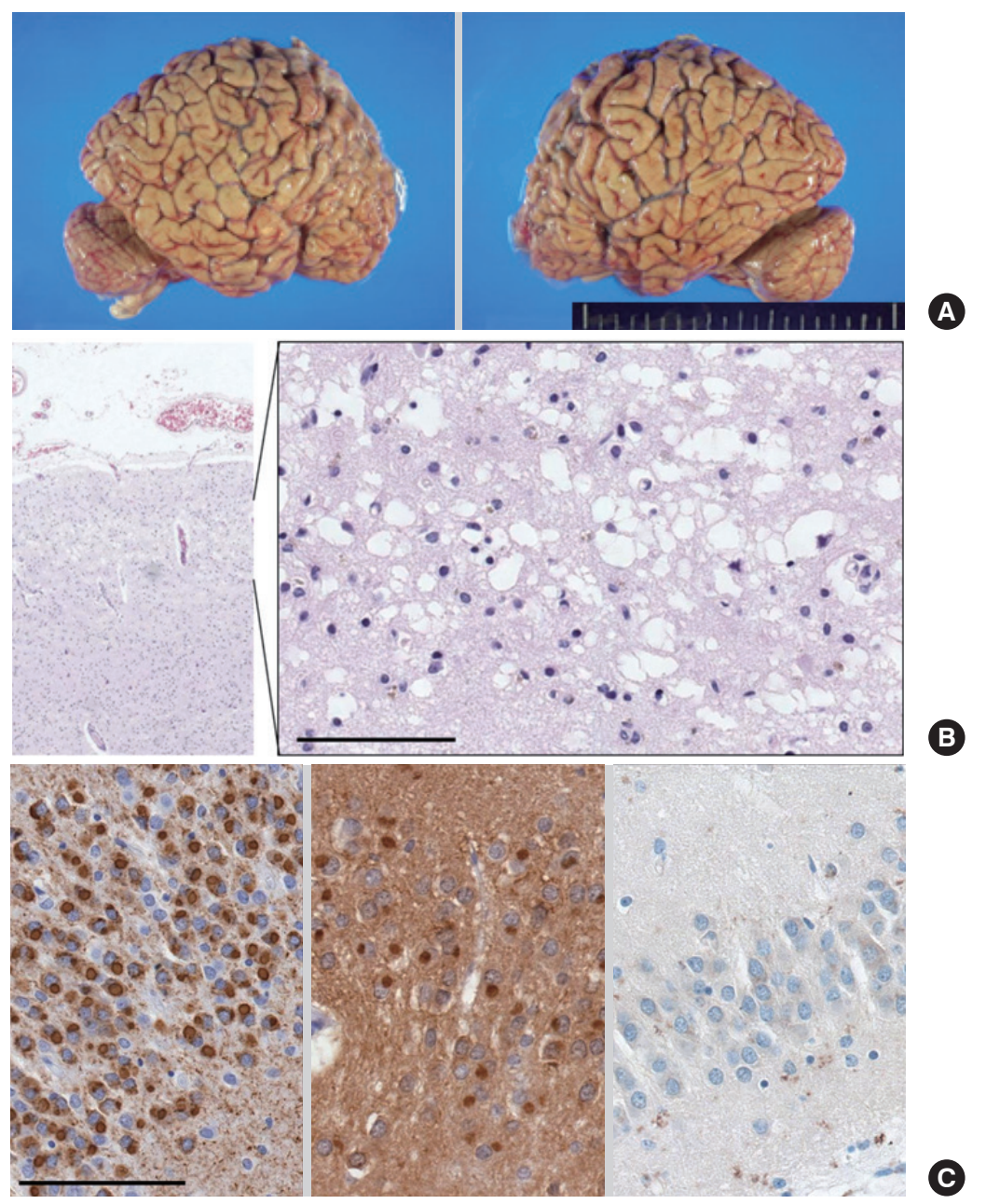

Fig. 2. Postmortem examination of a 63-year-old woman. (A) Severe gyral atrophy in bilateral frontal lobes with mild gyral atrophy of bilateral parietal and medial temporal lobes (scale bar $=10 \mathrm{~cm}$ ). (B) Microvacuolation, neuronal loss, and gliosis in frontal cortex layer 2 and 3 (H\&E stain, $\times$ 400; scale bar $=100 \mu \mathrm{m}$ ). (C) Tau-positive intracytoplasmic inclusion in dentate gyrus (Tau [left], 3-repeat Tau stain [mid], and 4-repeat Tau [right], $\times 400$; scale bar $=100 \mu \mathrm{m})$.

and a-synucleinopathy, a pathological diagnosis of FTLD-tau, PiD, was rendered.

\section{Case 2}

A 71-year-old right-handed man visited our clinic because of language dysfunction, left hand clumsiness, and memory impairment. He had started to show slow speech velocity, stuttering, and word-finding difficulty half a year prior to his first visit. There was no familial history of dementia. He was suffering from hypertension.

On neurological examination, the patient showed ideomotor apraxia and poor performance in fist-edge-palm. He also experienced bradykinesia in the left upper limb. He scored 25 out of 30 on MMSE. Detailed neuropsychological tests revealed that his attention, language, verbal memory, and frontal executive function, except visuospatial function and visual memory, were decreased. On WAB, he was overall fluent, but showed frequent hesitation, halting, repetition of syllables, phonemic paraphasia, and word-finding difficulty. Word comprehension, repetition, and naming were slightly decreased. These results indicate a mild degree of anomic aphasia (Table 1). His apolipoprotein E genotype was e3/e3. On FLAIR images, diffuse brain atrophy and periventricular white matter hyperintensities in the anterior and posterior portions were seen. On gradient echo images, strictly multiple lobar microbleeds and cortical superficial siderosis in the left parietal cortex were observed, suggesting probable cerebral amyloid angiopathy (CAA). [18F]-fluorodeoxyglucose positron emission tomography (FDG-PET) revealed moderate hypometabolism in the bilateral frontal cortex, which was more severe on the left side (Fig. 3). Based on his clinical symptoms, WAB, detailed neuropsychological tests, brain 

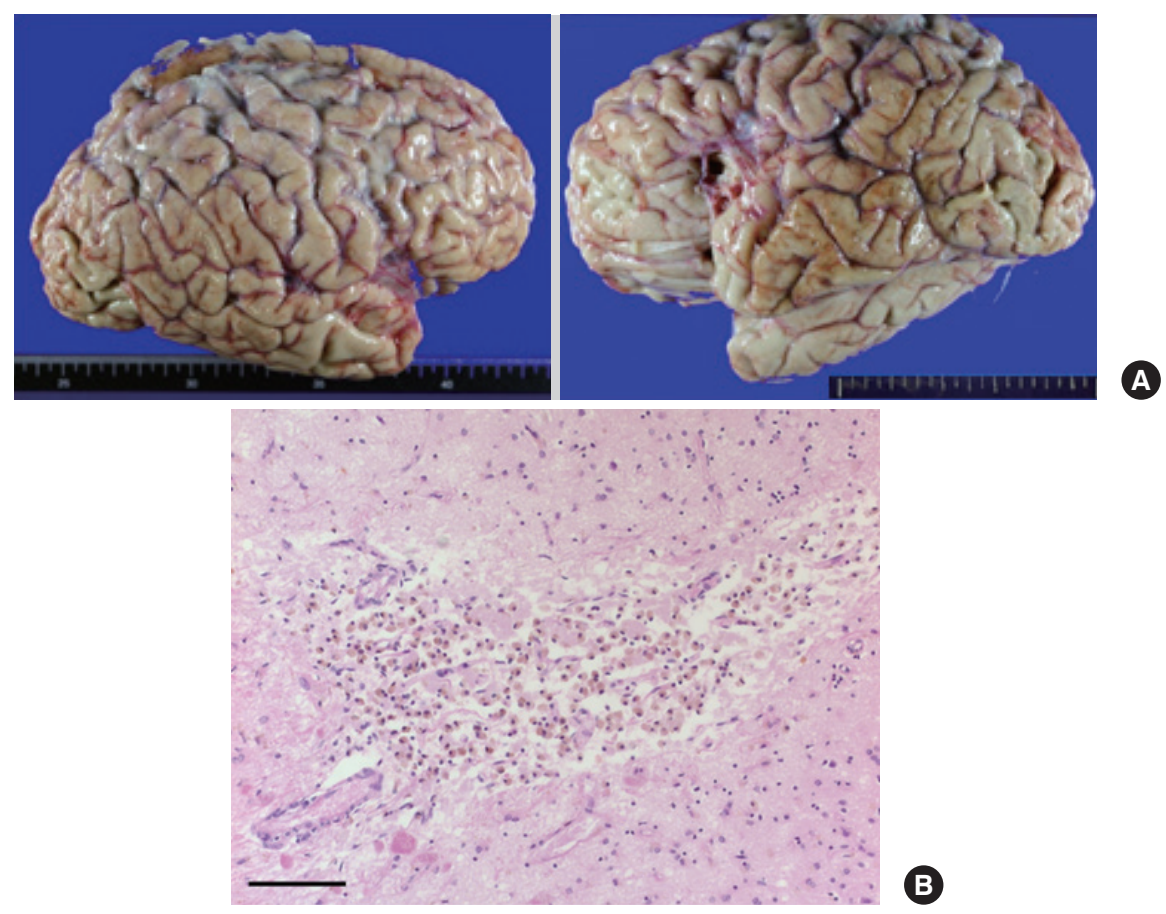

B

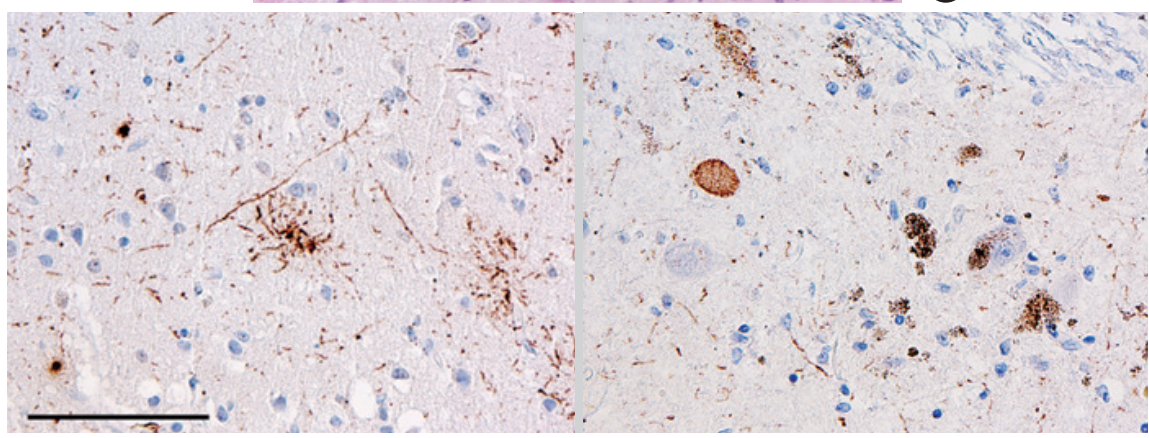

Fig. 3. Postmortem examination of a 71-year-old man. (A) Severe atrophy in bilateral precentral gyrus (scale bar $=10 \mathrm{~cm}$ ). (B) Remote microinfarct with hemosiderin-laden macrophages in temporal cortex (H\&E stain, $\times 100$; scale bar $=100 \mu \mathrm{m}$ ). (C) Tufted astrocyte in inferior parietal lobule (left), and globose tangles (arrow) in substantia nigra (right) (Tau stain, $\times 400$; scale bar $=100 \mu \mathrm{m}$ ).

MRI, and FDG-PET, the patient was clinically diagnosed with nfvPPA. Three years after his first visit, he showed cogwheel rigidity in the bilateral upper and lower limbs. However, the patient did not show axial rigidity, postural instability and vertical gaze limitation. The overall language function worsened, and he demonstrated phonemic paraphasia, neurogenic stuttering, and perseveration. He did not show dysarthria or apraxia of speech. These results indicate a moderate degree of anomic aphasia (Table 1). Four years after his first visit, he showed impatience and repetitive motor behaviors, such as tearing papers. Six years after his first visit, he was unable to walk anymore and died of pneumonia at the age of 77 years. After his death, his body underwent a brain autopsy at the Samsung Medical Center.

Postmortem examination showed a decreased brain weight of $940 \mathrm{~g}$ (standard for age: 1,300 g). Gross examination revealed marked atrophy of the bilateral precentral gyrus with moderate atrophy of the bilateral frontal and superior temporal gyri (Fig. 3A). On microscopic examination, tufted astrocytes were identified in the middle frontal gyrus, inferior parietal lobule, and occipital lobe. Globoid tangles and pretangles were observed in the temporal lobe, putamen, subthalamic nucleus, pons, and medulla oblongata (Fig. $3 \mathrm{C}$ ). $\beta$ -Amyloid plaques with amyloid angiopathy were present in the occipital cortex, amygdala, hippocampus, and cerebellum. Atherosclerosis in the vessels of the circle of Willis and hemosiderin pigmentation in the left inferior parietal lobule, suggestive of old subarachnoid hemorrhage, were noted. Microinfarcts were identified in the left frontal, temporal, and parietal lobes. Overall, the patient was diagnosed with FT- 
LD-tau, PSP with Alzheimer's disease (Braak Stage II), CAA, and cerebrovascualr disease.

\section{DISCUSSION}

Here, we report two clinically diagnosed nfvPPA cases. Both patients showed decreased fluency and effortful and decreased speech. As the disease progressed, they showed not only language dysfunction but also other symptoms, such as behavioral changes or motor dysfunction. However, they demonstrated showed several distinguished characteristics according to the pathological diagnosis. The patient pathologically diagnosed with PiD showed early age at onset and initial personality change accompanied by language dysfunction. On the other hand, the patient with PSP showed late age at onset and initial parkinsonian features accompanied by language dysfunction.

Patients with nfvPPA might show distinct clinical features depending on the underlying pathologies. Clinicopathological association between nfvPPA clinical syndrome and FTLD neuropathological diagnosis is not specific; nfvPPA is associated with multiple pathologies, including tauopathy, TDP type A pathology, and Alzheimer's disease pathology $[6,8]$. The first case was pathologically diagnosed as PiD. PiD, a rare subtype of FTLD-tau, is characterized by right dominant frontotemporal atrophy and clinical features of bvFTD. However, a few patients with PiD have showed left dominant frontal and peri-Sylvian atrophy and clinical features of nfvPPA. Gross neuropathological findings of PiD show circumscribed lobar atrophy, and microscopic findings are characterized by neuronal loss, gliosis, and argyrophilic and round intraneuronal inclusions (Pick's body). Pick's body is composed of a tau protein containing three microtubule-binding repeats (3R) [9].

PSP primarily presents with atypical parkinsonism with axial rigidity, frequent falls, postural instability, and progressive gaze palsy [9]. In particular, $80 \%$ to $90 \%$ of clinically suspected progressive supranuclear palsy syndrome (PSPS) show PSP pathology. However, approximately $10 \%$ of PSP presents the clinical features of nfvPPA, similar to our second case [6]. The cardinal neuropathological findings consisted of tufted astrocytes, globose neurofibrillary tangles, and oligodendroglial coiled bodies. These astrocytic, neuronal, and oligodendroglial lesions of PSP are mainly composed of tau protein containing four microtubule-binding repeats (4R) [9].

In conclusion, clinically diagnosed nfvPPA patients are expected to have various pathological diagnoses. Patients with nfvPPA might show distinct clinical features depending on the underlying pathologies.

\section{CONFLICTS OF INTEREST}

No potential conflict of interest relevant to this article was reported. Sang Won Seo has been editorial board of P\&FM since December 2017. He was not involved in the review process of this case report.

\section{ACKNOWLEDGMENTS}

This study was funded by the Research of Korea Centers for Disease Control and Prevention (2021-ER1003-00).

\section{ORCID}

Song Hwangbo https://orcid.org/0000-0002-7702-6036 Soohyun Hwang https://orcid.org/0000-0003-4581-3286 Mee Kyung Suh https://orcid.org/0000-0003-2558-8018 Seung Joo Kim https://orcid.org/0000-0001-9391-1767 Yeshin Kim https://orcid.org/0000-0002-4643-5478 Hee Jin Kim https://orcid.org/0000-0002-3186-9441 Duk L. Na https://orcid.org/0000-0002-0098-7592 Sang Won Seo https://orcid.org/0000-0002-8747-0122 Yeon-Lim Suh https://orcid.org/0000-0001-5809-2401

\section{AUTHOR CONTRIBUTIONS}

Conception or design: $\mathrm{SH}, \mathrm{SH}$.

Acquisition, analysis, or interpretation of data: $\mathrm{SH}, \mathrm{SH}, \mathrm{MKS}$, SJK, YK, HJK, DLN, SWS, YLS.

Drafting the work or revising: $\mathrm{SH}, \mathrm{SH}$.

Final approval of the manuscript: SH, SH, MKS, SJK, YK, HJK, DLN, SWS, YLS.

\section{REFERENCES}

1. Boeve BF, Lang AE, Litvan I. Corticobasal degeneration and its relationship to progressive supranuclear palsy and frontotemporal dementia. Ann Neurol 2003;54 Suppl 5:S15-9.

2. Josephs KA. Frontotemporal dementia and related disorders: deciphering the enigma. Ann Neurol 2008 Jul;64:414.

3. Arai T, Hasegawa M, Akiyama H, Ikeda K, Nonaka T, Mori H, et al. TDP-43 is a component of ubiquitin-positive tau-negative inclusions in frontotemporal lobar degeneration and 
amyotrophic lateral sclerosis. Biochem Biophys Res Commun 2006;351:602-11.

4. Neumann M, Sampathu DM, Kwong LK, Truax AC, Micsenyi MC, Chou TT, et al. Ubiquitinated TDP-43 in frontotemporal lobar degeneration and amyotrophic lateral sclerosis. Science 2006;314:130-3.

5. Knopman DS, Boeve BF, Parisi JE, Dickson DW, Smith GE, Ivnik RJ, et al. Antemortem diagnosis of frontotemporal lobar degeneration. Ann Neurol 2005;57:480-8.

6. Josephs KA, Petersen RC, Knopman DS, Boeve BF, Whitwell JL, Duffy JR, et al. Clinicopathologic analysis of frontotemporal and corticobasal degenerations and PSP.
Neurology 2006;66:41-8.

7. Forman MS, Farmer J, Johnson JK, Clark CM, Arnold SE, Coslett HB, et al. Frontotemporal dementia: clinicopathological correlations. Ann Neurol 2006;59:952-62.

8. Spinelli EG, Mandelli ML, Miller ZA, Santos-Santos MA, Wilson SM, Agosta F, et al. Typical and atypical pathology in primary progressive aphasia variants. Ann Neurol 2017; 81:430-43.

9. Dickson DW, Kouri N, Murray ME, Josephs KA. Neuropathology of frontotemporal lobar degeneration-tau (FTLD-tau). J Mol Neurosci 2011;45:384-9. 